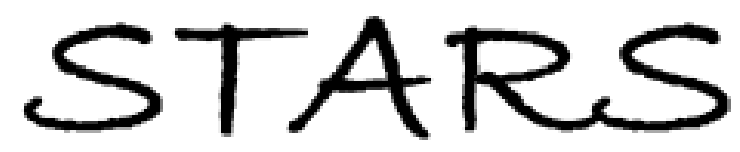

University of Central Florida

STARS

$1-1-2005$

\title{
Numerical model of the temperature dependence of the up- conversion efficiency of fluoride crystals codoped with ytterbium and thulium
}

\author{
A. Rapaport \\ University of Central Florida \\ J. Milliez \\ University of Central Florida \\ M. Bass \\ University of Central Florida
}

\author{
A. Cassanho \\ H. Jenssen \\ Find similar works at: https://stars.library.ucf.edu/facultybib2000 \\ University of Central Florida Libraries http://library.ucf.edu
}

This Article is brought to you for free and open access by the Faculty Bibliography at STARS. It has been accepted for inclusion in Faculty Bibliography 2000s by an authorized administrator of STARS. For more information, please contactSTARS@ucf.edu.

\section{Recommended Citation}

Rapaport, A.; Milliez, J.; Bass, M.; Cassanho, A.; and Jenssen, H., "Numerical model of the temperature dependence of the up-conversion efficiency of fluoride crystals codoped with ytterbium and thulium" (2005). Faculty Bibliography 2000s. 5571.

https://stars.library.ucf.edu/facultybib2000/5571

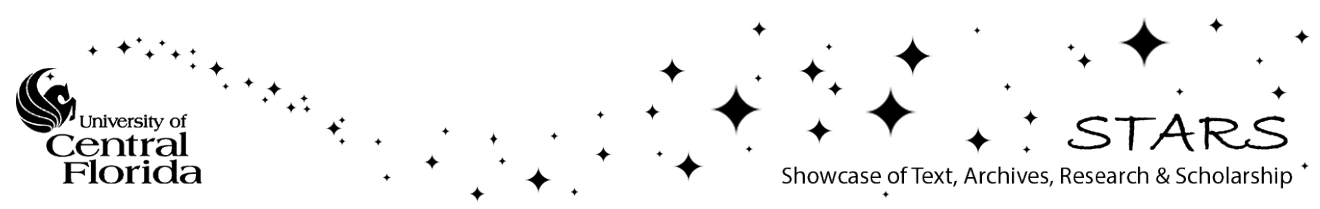




\section{Numerical model of the temperature dependence of the up-conversion efficiency of fluoride crystals codoped with ytterbium and thulium}

Cite as: J. Appl. Phys. 97, 123527 (2005); https://doi.org/10.1063/1.1939066

Submitted: 10 February 2005 . Accepted: 25 April 2005 . Published Online: 22 June 2005

A. Rapaport, J. Milliez, M. Bass, A. Cassanho, and H. Jenssen

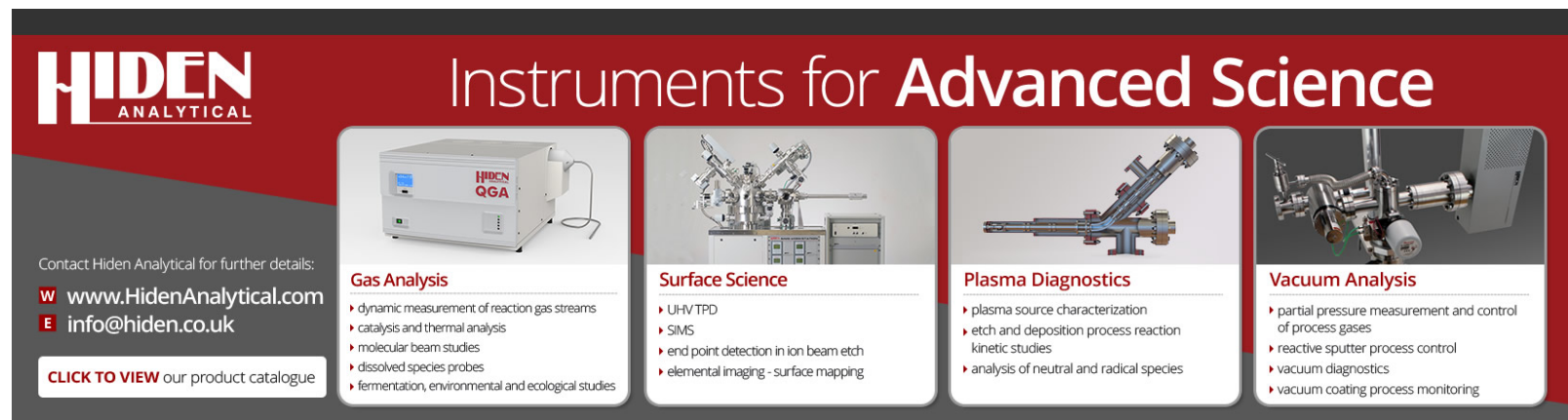




\title{
Numerical model of the temperature dependence of the up-conversion efficiency of fluoride crystals codoped with ytterbium and thulium
}

\author{
A. Rapaport, ${ }^{a}$ J. Milliez, and M. Bass \\ College of Optics and Photonics/Center for Research and Education in Optics and Lasers (CREOL)/Florida \\ Photonics Center of Excellence (FPCE), University of Central Florida, Orlando, Florida 32816
}

\author{
A. Cassanho and $\mathrm{H}$. Jenssen \\ AC Materials, Orlando, Florida 32792
}

(Received 10 February 2005; accepted 25 April 2005; published online 22 June 2005)

\begin{abstract}
We review the role of temperature on the up-conversion efficiency of materials that are good candidates to be used as the three primary-color emitters in optically written displays. A rate equation model is given for the blue emitting material that includes temperature-dependent coefficients for energy transfer and cross relaxation. Increased temperature leads to decreased up-conversion efficiency. The effect of pumping conditions on heating the emitting material is illustrated and explained. Steps to reduce the temperature rise when pumping up-conversion materials are described. (C) 2005 American Institute of Physics. [DOI: 10.1063/1.1939066]
\end{abstract}

\section{INTRODUCTION}

The concept of frequency up-conversion (UC) of infrared-to-visible light in rare-earth (RE)-doped materials was reported more than 40 years ago. ${ }^{1}$ The efficiency that was observed or expected for this process was low in singly doped media, but it was quickly noticed that the mechanism could be made one or two orders of magnitude more efficient by using ytterbium $\left(\mathrm{Yb}^{3+}\right)$ as a sensitizer ion in addition to the active ion: erbium $\left(\mathrm{Er}^{3+}\right)$, holmium $\left(\mathrm{Ho}^{3+}\right)$, or thulium $\left(\mathrm{Tm}^{3+}\right){ }^{2-7}$ Efficient UC materials were extensively investigated as they could be used for several potentially important photonic applications including UC lasers (visible lasers that are pumped by infrared diode lasers) and displays. However, because no powerful source existed in the 980-nm region in order to excite such up converters no practical product came out of the research. With the development of powerful 980-nm diode lasers for the telecommunication industry this technology that appeared to be too inefficient in the past now has legitimate practical applications. Nevertheless, efficiency continues to limit UC emitters' applicability to display technology. Therefore every technological step that can improve UC efficiencies must be considered.

First, it is worth noting some of the attractive features of an optically excited UC display. Displays based on the UC emitters that we have studied (e.g., fluoride crystals with $\mathrm{Yb}^{3+}$ codoped with another rare-earth dopant ion) are scalable: the materials are ground into powder and dispersed in a polymeric host (phosphorylated polymethylmethacrylate or $p$-PMMA). The preparation is then cast on a substrate and an infrared laser beam is scanned across the screen which does not have any inherent size limitation. In addition, no high voltage or vacuum tube is needed and the optical path can be folded in order to reduce the depth of the display. The lowenergy pump photons used do not produce damage in the up-conversion materials so the screens do not suffer perfor-

\footnotetext{
a) Author to whom correspondence should be addressed; electronic mail: rapaport@creol.ucf.edu
}

mance deterioration as do down-conversion displays (plasma display screens). The strongest advantages of such displays remain that they are emissive displays that can achieve very high brightness (tens of $\mathrm{kcd} / \mathrm{m}^{2}$ in the three primary colors) without damage to the emitters and they offer very saturated colors because of the wide color gamut covered by the three main emission lines.

In this paper, we expand on the results reported in Ref. 8 and present the numerical model for the acceptor-donor systems we have studied. We include the temperature dependence of the energy-transfer rates in the rate equations model, as well as the temperature changes caused by the various energy transfers. We compare the results of the simulation for various pump durations and dopant concentrations to the experimental results obtained for a blue emitting UC material: $\mathrm{Yb}^{3+}, \mathrm{Tm}^{3+}$-doped yttrium lithium fluoride or Yb,Tm:YLF. The experimental results for our red $\left(\mathrm{Yb}^{3+}, \mathrm{Er}^{3+}\right.$-doped yttrium fluoride or $\left.\mathrm{Yb}, \mathrm{Er}: \mathrm{YF}_{3}\right)$ and green $\left(\mathrm{Yb}^{3+}, \mathrm{Er}^{3+}\right.$-doped sodium yttrium fluoride or $\left.\mathrm{Yb}, \mathrm{Er}: \mathrm{NYF}\right)$ are also presented. ${ }^{9-12}$ We then describe the improvements that result when using a heat conductive substrate that allows some control of the emitters' temperature.

\section{RESULTS}

With today's mathematical software it is possible to solve systems of coupled nonlinear differential equations numerically. We describe the model developed for the blue emitter, Yb,Tm:YLF which shows the most dramatic dependence on emitter temperature. This material can be described by the energy-level diagram in Fig. 1. In order to avoid unnecessary complication, we do not include the ${ }^{1} D_{2}$ level of $\mathrm{Tm}^{3+}$ in our treatment of the system as it does not help to illustrate the problem. The set of rate equations that corresponds to Fig. 1 ignoring the ${ }^{1} D_{2}$ state is, including the appropriate energy-transfer, back-transfer, cross-relaxation, and spontaneous emission rates: 


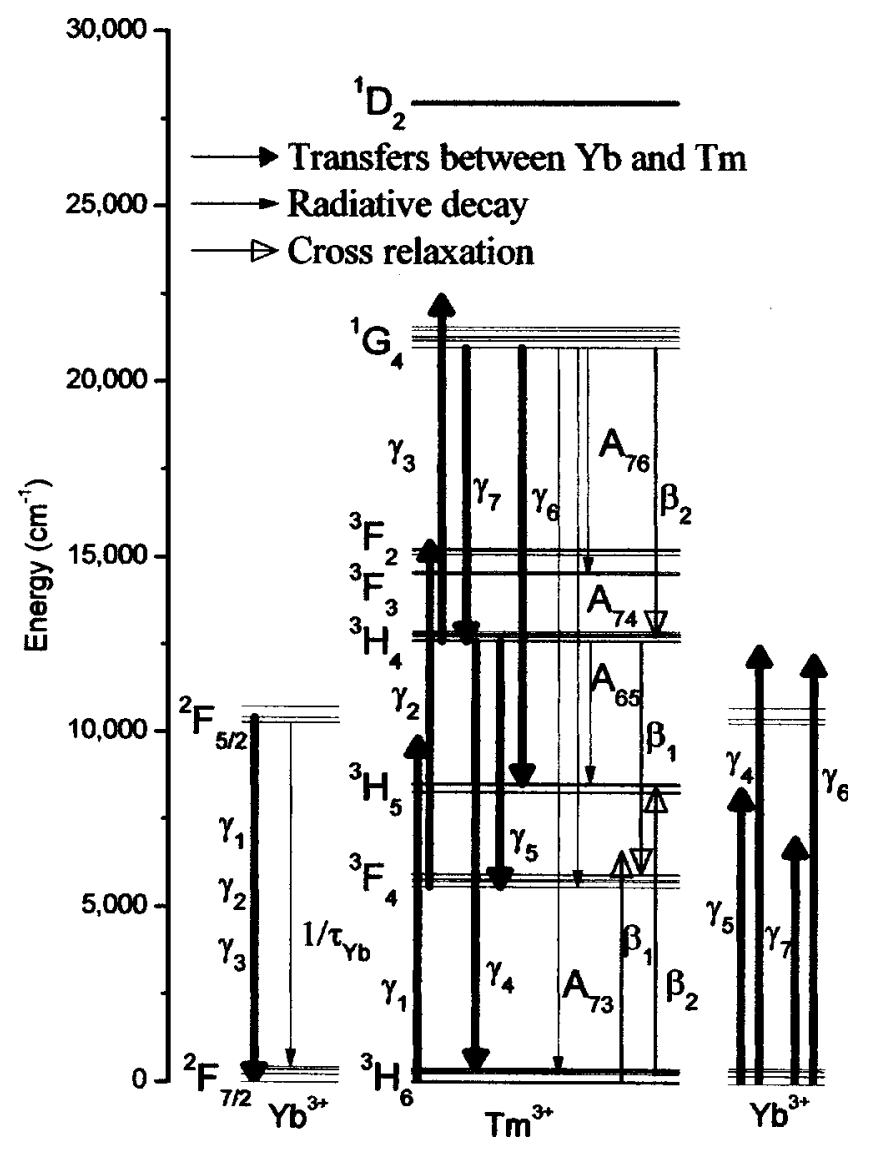

FIG. 1. Energy-level diagram of $\mathrm{Yb}^{3+}$ and $\mathrm{Tm}^{3+}$ in YLF. Possible energy transfers from $\mathrm{Yb}^{3+}$ to $\mathrm{Tm}^{3+}$ are indicated in thick solid lines, radiative decays are indicated in thin solid lines with closed arrows, and cross relaxations are indicated in thin solid lines with opened arrows. Energy back transfer from $\mathrm{Tm}^{3+}$ to $\mathrm{Yb}^{3+}$ is also possible along the same pathways.

$$
\begin{aligned}
\dot{n}_{1}= & \frac{n_{2}}{\tau_{\mathrm{Yb}}}-\sigma_{\mathrm{Yb}}^{\text {abs }} \frac{I_{\mathrm{pump}}[t]}{h \nu_{\mathrm{pump}}}\left(n_{1}-n_{2}\right)+\gamma_{1}[T] n_{2} n_{3}+\gamma_{2} n_{2} n_{4} \\
& +\gamma_{3}[T] n_{2} n_{6}-\gamma_{4}[T] n_{1} n_{6}-\gamma_{5}[T] n_{1} n_{6}-\gamma_{6}[T] n_{1} n_{7} \\
& -\gamma_{7}[T] n_{7} n_{1},
\end{aligned}
$$$$
\dot{n}_{2}=-\dot{n}_{1},
$$$$
\dot{n}_{3}=\frac{n_{4}}{\tau_{4}}+A_{63} n_{6}+A_{73} n_{7}-\gamma_{1}[T] n_{2} n_{3}+\gamma_{4}[T] n_{1} n_{6}
$$$$
-\beta_{1}[T] n_{3} n_{6}-\beta_{2}[T] n_{3} n_{7},
$$$$
\dot{n}_{4}=-\frac{n_{4}}{\tau_{4}}+\frac{n_{5}}{\tau_{5}}+A_{64} n_{6}+A_{74} n_{7}-\gamma_{2} n_{2} n_{4}+\gamma_{5}[T] n_{1} n_{6}
$$$$
+2 \beta_{1}[T] n_{3} n_{6}+\beta_{2}[T] n_{3} n_{7},
$$$$
\dot{n}_{5}=-\frac{n_{5}}{\tau_{5}}+A_{65} n_{6}+A_{75} n_{7}+\gamma_{1}[T] n_{2} n_{3}+\gamma_{6}[T] n_{1} n_{7},
$$$$
\dot{n}_{6}=-\frac{n_{6}}{\tau_{6}}+A_{76} n_{7}+\gamma_{2} n_{2} n_{4}-\gamma_{3}[T] n_{2} n_{6}-\gamma_{4}[T] n_{1} n_{6}
$$$$
-\gamma_{5}[T] n_{1} n_{6}+\gamma_{7}[T] n_{1} n_{7}-\beta_{1}[T] n_{3} n_{6}+\beta_{2}[T] n_{3} n_{7},
$$

$$
\begin{aligned}
\dot{n}_{7}= & -\frac{n_{7}}{\tau_{7}}+\gamma_{3}[T] n_{2} n_{6}-\gamma_{6}[T] n_{1} n_{7}-\gamma_{7}[T] n_{7} n_{1} \\
& -\beta_{2}[T] n_{3} n_{7} .
\end{aligned}
$$

Room-temperature values of the various coefficients are given in Table I. The energy levels indicated in Fig. 1 allow one to calculate the energy mismatch for each energy transfer, back transfer, and cross relaxation. The number of phonons $p$ needed to bridge that gap determines the temperature dependence of the rate associated with that process (given in Table I for each transition). The effective phonon energy is taken at $460 \mathrm{~cm}^{-1}$ for YLF. ${ }^{13}$ Two cases arise: if there is emission of phonons during the process, the rate has the functional form ${ }^{14}$

$$
\gamma[T]=\gamma\left[T_{0}\right]\left(\frac{\bar{n}[T]+1}{\bar{n}\left[T_{0}\right]+1}\right)^{p},
$$

where $\bar{n}[T]=1 / \exp \left[\Delta E / k\left(T_{0}+\Delta T[\mathrm{t}]\right)\right]-1$ is the occupation number for the effective phonons of energy $\Delta E=460 \mathrm{~cm}^{-1}$, and $k$ is Boltzmann's constant.

If there is absorption of phonons during the process, the functional form of the rate is ${ }^{14}$

$$
\gamma[T]=\gamma\left[T_{0}\right]\left(\frac{\bar{n}[T]}{\bar{n}\left[T_{0}\right]}\right)^{p} .
$$

The rates affected by temperature change are noted as dependent on the temperature $T$ in Eq. (1). Note that the transfer to ${ }^{3} \mathrm{H}_{5}$ is resonant, so $\gamma_{2}$ does not vary with temperature. However, the transfer is followed by a nonradiative decay that has to be taken into account when calculating the temperature change.

A few other differential equations are needed in order to solve Eqs. (1). First, we need to follow the evolution of the temperature as the material is being pumped. Each phonon being absorbed or emitted changes the energy inside the lattice, and so changes the local temperature. The change of temperature is taken to be proportional to the total change in phonon density. We also take into account some heat diffusion inside the material, so that the temperature variation obeys

$$
\begin{aligned}
\dot{T} \propto & p_{\gamma_{1}} \gamma_{1}[T] n_{2} n_{3}+p_{\gamma_{2}} \gamma_{2} n_{2} n_{4}+p_{\gamma_{3}} \gamma_{3}[T] n_{2} n_{6} \\
& +p_{\gamma_{4}} \gamma_{4}[T] n_{1} n_{6}+p_{\gamma_{5}} \gamma_{5}[T] n_{1} n_{6}+p_{\gamma_{6}} \gamma_{6}[T] n_{1} n_{7} \\
& +p_{\gamma_{7}} \gamma_{7}[T] n_{1} n_{7}-\frac{T}{t_{D}}
\end{aligned}
$$

where $t_{D}$ is a time characteristic of heat diffusivity through the heated region. Finally, the visible fluorescence emission rate is given by the simple equation

$$
\dot{S}_{\text {blue }}=A_{73} n_{7}=\beta_{73} \frac{n_{7}}{\tau_{7}}
$$

and we define the efficiency of the up-conversion process as 
TABLE I. Room-temperature values of the parameters used in the system of Eq. (1).

\begin{tabular}{|c|c|c|c|}
\hline Coefficient & Transition & Value used & $\begin{array}{l}\text { Number of phonons } p \text { involved } \\
(+ \text { for emission, }- \text { for absorption })\end{array}$ \\
\hline \multicolumn{4}{|c|}{$\begin{array}{l}2 \mathrm{~ms}^{\mathrm{a}} \\
3.3 \times 10^{-21} \mathrm{~cm}^{2 \mathrm{a}}\end{array}$} \\
\hline \multicolumn{4}{|c|}{$3.3 \times 10^{-21} \mathrm{~cm}^{2 \mathrm{a}}$} \\
\hline$\tau_{4}$ & ${ }^{3} F_{4} \rightarrow{ }^{3} H_{6}$ & $15 \mathrm{~ms}^{\mathrm{a}}$ & \\
\hline \multicolumn{4}{|c|}{${ }^{3} H_{5} \rightarrow{ }^{3} F_{4}$} \\
\hline \multicolumn{4}{|c|}{$0.9032 / \tau_{6}{ }^{b}$} \\
\hline \multicolumn{4}{|c|}{$0.0697 / \tau_{6}^{\mathrm{b}}$} \\
\hline \multicolumn{4}{|c|}{$0.0271 / \tau_{6}^{\mathrm{b}}$} \\
\hline \multicolumn{2}{|l|}{$\tau_{6}$} & $2.258 \mathrm{~ms}^{\mathrm{b}}$ & \\
\hline$A_{73}$ & ${ }^{1} G_{4} \rightarrow{ }^{3} H_{6}$ & $0.51 / \tau_{7}^{\mathrm{b}}$ & \\
\hline \multicolumn{4}{|c|}{${ }^{1} G_{4} \rightarrow{ }^{3} F_{4}$} \\
\hline \multicolumn{4}{|c|}{${ }^{1} G_{4} \rightarrow{ }^{3} H_{5}$} \\
\hline \multicolumn{4}{|c|}{${ }^{1} G_{4} \rightarrow{ }^{3} H_{4}$} \\
\hline \multicolumn{4}{|c|}{$1.158 \mathrm{~ms}^{\mathrm{b}}$} \\
\hline \multicolumn{4}{|c|}{${ }^{2} F_{5 / 2} \rightarrow{ }^{2} F_{7 / 2}$ while ${ }^{3} H_{3} \rightarrow{ }^{3} H_{5}$} \\
\hline \multicolumn{4}{|c|}{${ }^{2} F_{5 / 2} \rightarrow{ }^{2} F_{7 / 2}$ while ${ }^{3} F_{4} \rightarrow{ }^{3} H_{4}$} \\
\hline \multicolumn{4}{|c|}{${ }^{2} F_{5 / 2} \rightarrow{ }^{2} F_{7 / 2}$ while ${ }^{3} H_{4} \rightarrow{ }^{1} G_{4}$} \\
\hline \multicolumn{4}{|c|}{${ }^{3} H_{4} \rightarrow{ }^{3} H_{6}$ while ${ }^{2} F_{7 / 2} \rightarrow{ }^{2} F_{5 / 2}$} \\
\hline \multicolumn{4}{|c|}{${ }^{3} H_{4} \rightarrow{ }^{3} F_{4}$ while ${ }^{2} F_{7 / 2} \rightarrow{ }^{2} F_{5 / 2}$} \\
\hline \multicolumn{4}{|c|}{${ }^{1} G_{4} \rightarrow{ }^{3} H_{5}$ while ${ }^{2} F_{7 / 2} \rightarrow{ }^{2} F_{5 / 2}$} \\
\hline \multicolumn{4}{|c|}{${ }^{1} G_{4} \rightarrow{ }^{3} H_{4}$ while ${ }^{2} F_{7 / 2} \rightarrow{ }^{2} F_{5 / 2}$} \\
\hline \multicolumn{4}{|c|}{${ }^{3} H_{6} \rightarrow{ }^{3} F_{4}$ while ${ }^{3} H_{4} \rightarrow{ }^{3} F_{4}$} \\
\hline \multicolumn{4}{|c|}{${ }^{3} H_{6} \rightarrow{ }^{3} H_{5}$ while ${ }^{1} G_{4} \rightarrow{ }^{3} H_{4}$} \\
\hline $\begin{array}{l}\mathrm{N}_{\text {at. }}=1.37 \times 1 \\
{ }^{\mathrm{a}} \text { From Ref. } 2 \\
{ }^{\mathrm{b}} \text { From Ref. } 2 \\
{ }^{\mathrm{c}} \text { From Ref. } 2 \\
{ }^{\mathrm{d}} \text { Estimated. }\end{array}$ & $\mathrm{cm}^{-3}$. & & \\
\hline
\end{tabular}

$$
\operatorname{Eff}=\frac{\int_{0}^{t_{\text {final }}} \dot{S}_{\text {blue }} d t}{\int_{0}^{t_{\text {final }}} I_{\text {pump }}(t) d t}
$$

Solving the rate equations numerically and computing the UC efficiency according to Eq. (6) leads to the calculated efficiencies shown in Fig. 2(a) for $0.4 \% \mathrm{Tm}, 25 \% \mathrm{Yb}$ :YLF for five different types of excitation: continuous, and pulsed with pump durations of $5 \mathrm{~ms}, 2.6 \mathrm{~ms}, 630 \mu \mathrm{s}$, and $350 \mu \mathrm{s}$. For comparison, Fig. 2(b) shows experimental results obtained for the same set of relative incident average powers and pump durations (the pulsed data were taken at $30-\mathrm{Hz}$ repetition frequency). In these experiments, the visible output emitted by a small volume of dry powdered Yb,Tm:YLF was collected with an 80-mm-diameter integrating sphere, fiber coupled to a calibrated compact array spectrometer (CAS140) by Instrument Systems. The excitation source was a 959-nm, cw diode laser beam and a rotating chopper with different slit widths was used to obtain varying pulse duration at $30 \mathrm{~Hz}$ (see also Ref. 15). These experimental results in Fig. 2(b) cannot be reproduced by a model that uses only temperature-independent coefficients. Our model is necessary to explain the data and we can conclude that pump duration and temperature play a crucial role in the UC performance.

In order to obtain the close agreement between numerical results and experimental data shown in Fig. 2, the time characteristic of heat diffusivity in the model was chosen to be $8 \mathrm{~ms}$. We can calculate the thermal diffusivity in YLF (Ref. 16) from

$$
\kappa=\frac{K}{\rho C}=0.019 \mathrm{~cm}^{2} \mathrm{~s}^{-1},
$$

where $K$ is the thermal conductivity $\left[0.06 \mathrm{~W} \mathrm{~cm}^{-1} \mathrm{~K}^{-1}\right.$ in YLF (Ref. 17)], $\rho$ is the material density $\left(3.95 \mathrm{~g} \mathrm{~cm}^{-3}\right)$, and $C$ is the heat capacity $\left(0.79 \mathrm{~J} \mathrm{~g}^{-1} \mathrm{~K}^{-1}\right)$. Using the relation $d$ $=\left(\kappa t_{D}\right)^{1 / 2}$ and where $t_{D}=8 \mathrm{~ms}$ as obtained from the model, we obtain a distance for heat diffusion of $125 \mu \mathrm{m}$. This result is consistent with the size of the sample and the absorption depth of the pump beam.

In order to evaluate the effect of temperature on the upconversion process efficiency, we performed an experiment where a hot plate and thermocouple were used to externally set the temperature, and a low-energy, 10-Hz, 4-ns pulse at $959 \mathrm{~nm}$ was used to provide UC excitation without affecting the temperature. The specific pump wavelength was chosen according to the results from a separate excitation spectrum measurement. ${ }^{9}$ The total blue up-conversion fluorescence was recorded and the drop in normalized up-conversion efficiency with increasing temperature is illustrated in Fig. 3. The same experiment was performed with the green and red emitters with the appropriate excitation wavelength and monitored fluorescence wavelength, and the results are also 

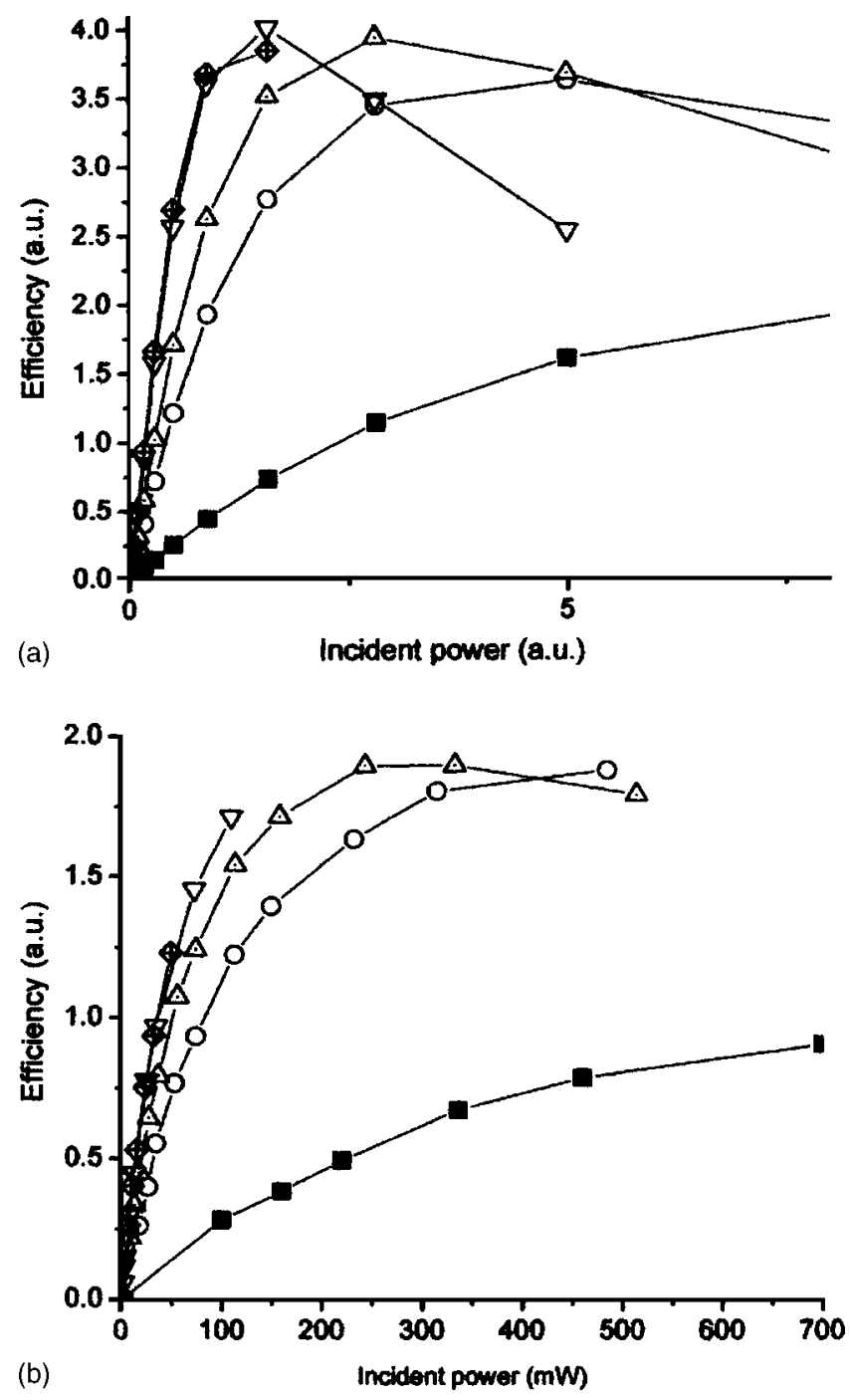

FIG. 2. (a) Calculated output power up-conversion efficiency in $0.4 \% \mathrm{Tm}$, $25 \% \mathrm{Yb}$ :YLF for five different types of excitation: continuous, and pulsed at $30 \mathrm{~Hz}$ with pump durations of $5 \mathrm{~ms}$ (circles), $2.6 \mathrm{~ms}$ (up triangles), $630 \mu \mathrm{s}$ (down triangles), and $350 \mu$ s (diamonds). The rate of heat diffusion is set to $8 \mathrm{~ms}$ in the calculations. (b) Experimentally measured output power upconversion efficiency in $0.4 \% \mathrm{Tm}, 25 \% \mathrm{Yb}$ :YLF for five different types of excitation: continuous, and pulsed at $30 \mathrm{~Hz}$ with pump durations of $5 \mathrm{~ms}$ (circles), $2.6 \mathrm{~ms}$ (up triangles), $630 \mu \mathrm{s}$ (down triangles), and $350 \mu \mathrm{s}$ (diamonds).

plotted in Fig. 3. These data confirm that temperature affects the up-conversion process and increased temperature reduces the UC efficiency of our materials.

We now evaluate the temperature of the up converters as they are operated and when the incident intensity is increased. This is done for various pump durations and when the powder was inside either an acrylic or a copper holder. The emission spectrum was recorded for each pumping condition. Figure 4 presents typical spectra in the blue obtained with the acrylic holder for two pumping conditions: $\mathrm{cw}$ and $30-\mathrm{Hz}, 2-\mathrm{ms}$ pulsed excitation. The output power was the same $(58 \mathrm{mlm})$ in both pumping conditions.

There are two emission bands near 450 and near $483 \mathrm{~nm}$. The emission band centered at $450 \mathrm{~nm}$ originates from the ${ }^{1} D_{2}$ level of Tm, which lies $7000 \mathrm{~cm}^{-1}$ above ${ }^{1} G_{4}$. These two levels are not thermally coupled but their emis-

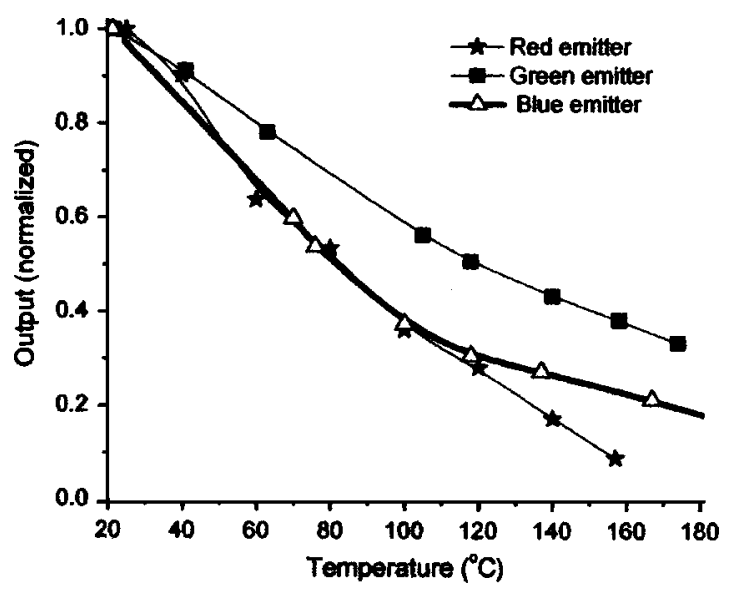

FIG. 3. Normalized output power at low incident pump intensity from the red (thin line and solid stars), green (thin line and solid squares), and blue (thick line and hollow triangles) up-conversion materials as a function of temperature.

sion spectra overlap. Therefore, to correctly estimate the spectral distribution of the 480-nm band as a function of temperature, one has to first subtract the tail of the emission band at $450 \mathrm{~nm}$. The resulting spectrum still has several emission peaks. We are particularly interested in the transitions at 465.5 and at $483 \mathrm{~nm}$ in Yb, Tm:YLF. They both originate from the same energy level ${ }^{1} G_{4}$ but from different Stark split sublevels. Those sublevels are thermalized and the spectral distribution is therefore a signature of the temperature inside the sample (fluorescence intensity ratio or FIR technique ${ }^{18,19}$ ). When the ratio of the emission at 465.5 to that at $483 \mathrm{~nm}$ is observed to increase, it means that the temperature of the emitting powder has increased. Figure 4 shows that for the same output luminance, the temperature of the sample is much higher when continuous pumping is used than when pulsed excitation is used.

We can map the ratio of the emission lines intensity to the temperature of the emitting powder using the measured emission spectra obtained at temperatures between 21 and $180^{\circ} \mathrm{C}$ set externally with the hot plate. Using those ratios, we determined the temperature of the emitting powder for various pumping conditions $(\mathrm{cw}$, and $30-\mathrm{Hz}, 5$ and $2-\mathrm{ms}$ pulses) and for two types of powder holders (acrylic and copper). The holders were $1-\mathrm{cm}$-long, 2.5 -cm-diameter cylinders with a 500- $\mu \mathrm{m}$-deep, $750-\mu \mathrm{m}$-diameter hole drilled at the center of a face. The temperatures are shown in Fig. 5(a), and the resulting measured efficiencies are given in Fig. 5(b). Several observations can be made from the temperature plot. First, for a given material and sample holder, the temperature in the powder depends only on the incident infrared power. This can be explained by the fact that the blue up-conversion process is about $4 \%$ or $5 \%$ efficient. Thus, a large part of the absorbed power is not reradiated (in the blue, red, or infrared) but is diffused nonradiatively inside the material through the emission of phonons which raise the temperature of the sample. Second, the results in the acrylic holder show that the emitting powder can reach very high temperatures with cw or even long-pulse duration excitation. This is because when using long-pulse duration excitation, the peak population densities are lower than when using short-pulse duration 


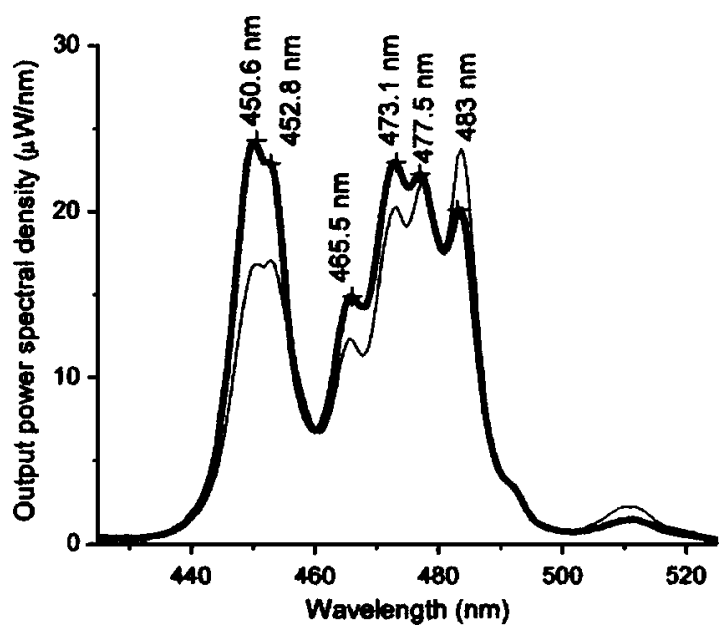

FIG. 4. Emission spectra of $0.4 \% \mathrm{Tm}, 25 \% \mathrm{Yb}$ :YLF. Two conditions of excitation were used: the bold line corresponds to continuous pumping; the thin line represents 2-ms duration pulse pumping at a repetition rate of $30 \mathrm{~Hz}$. The output luminance in the blue was the same in both excitation conditions $(58 \mathrm{mlm})$. The sample holder was made of acrylic.

excitation for the same average incident power. As a result, the transfer rate probabilities (proportional to the population densities in the initial levels) are lower in the long duration pulse case and the up-conversion efficiency is less [see Fig. 5(b)]. In order to achieve the same population densities, the average power required is higher using long-pulse duration excitation. This results in higher temperature, and consequently, in loss of efficiency for the up-conversion process, as shown in Fig. 3. The lower temperatures attained when using short-pulse duration excitation explain the higher efficiencies obtained in Fig. 5(b). This improvement in efficiency is verified down to excitation pulses of the order of the transfer time from $\mathrm{Yb}^{3+}$ to $\mathrm{Tm}^{3+}$. For pulses shorter than this transfer time, no improvement can be seen as the energy remains stored in the $\mathrm{Yb}$ excited state. Finally, it is straightforward to reduce the temperature of the emitting powder by simply using a high thermal diffusivity sample holder. By simply using a copper holder, we achieved significant heat extraction resulting in lower emitter temperatures and, as a result, higher efficiencies were detected. Using smaller holes for pixels and designing the holes in order to maximize the thermal contact between powder and substrate should help in operating the powder close to room temperature, and therefore achieving still higher efficiencies.

The results obtained for the red and green up-conversion emitters are very similar. For the green emitter, the upconversion process requires only two resonant steps instead of three steps with high-energy mismatch for the blue emitter. Therefore the incident power densities required to attain saturation of the efficiency are smaller and there is less heating of the powder for the same incident power. As a result, after analysis of the emission spectra in various excitation conditions, temperatures of only $10-20{ }^{\circ} \mathrm{C}$ above room temperature are observed when using the acrylic sample holder with a 1-mm-diameter hole, even for continuous excitation. This rise in temperature is not sufficient to significantly alter the up-conversion efficiency for the green emitters. For the red emitter, a third up-conversion step contributes to the red
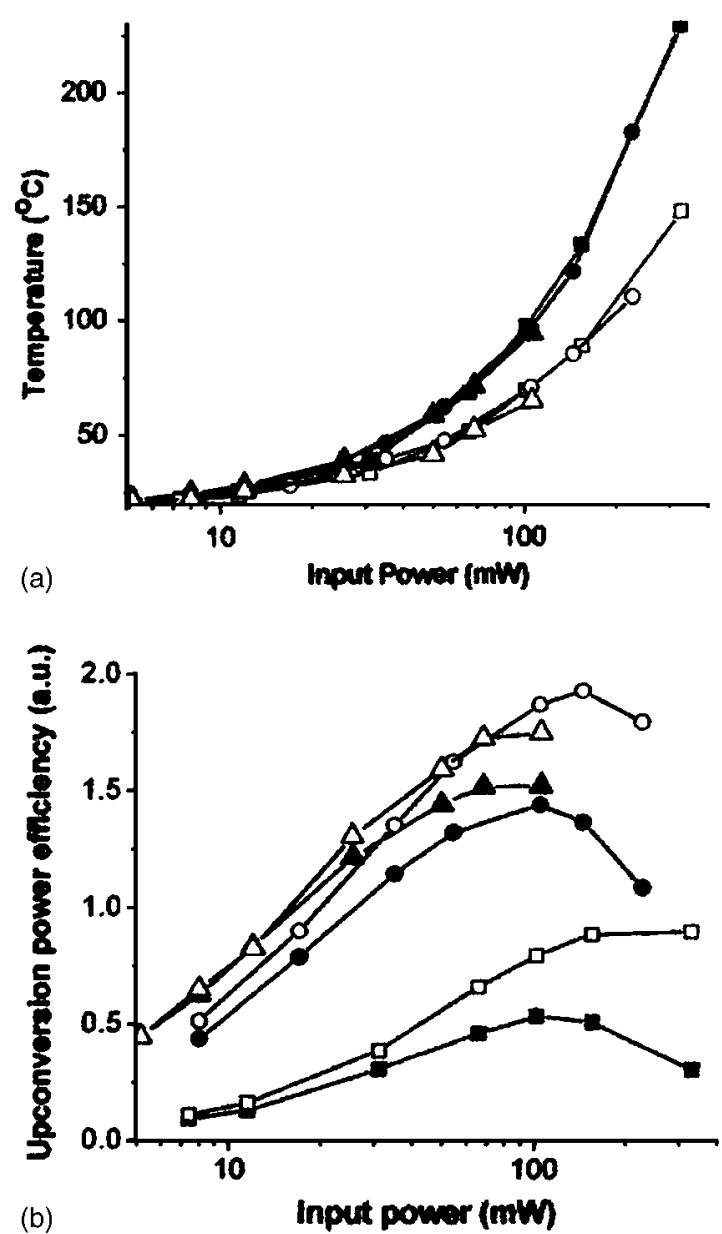

FIG. 5. Up-conversion temperature and efficiency of a sample of $0.4 \% \mathrm{Tm}$, $25 \% \mathrm{Yb}$ :YLF. The solid symbols correspond to an acrylic sample holder and the open symbols are for a copper sample holder with the hole containing the powder with a $750-\mu \mathrm{m}$-diameter, $500-\mu \mathrm{m}$-deep cylinder. The squares are for $\mathrm{cw}$ excitation, the circles are for $30 \mathrm{~Hz}, 5$-ms duration pulses, and the triangles are for $30-\mathrm{Hz}, 2-\mathrm{ms}$ duration pulses. The excitation wavelength was $959 \mathrm{~nm}$. (a) Temperature of the emitting powder. (b) Relative efficiency measured.

emission especially at higher incident power densities. ${ }^{20}$ However, the energy mismatch and nonradiative decays are much less than during the blue up-conversion process, resulting once again in less heating of the material, and an observed rise in temperature of less than $20^{\circ} \mathrm{C}$. In a full color screen the temperature would have to be controlled due to heating by the blue emitter.

Finally, we have measured the absolute efficiencies for our best performing, three primary-colors up-conversion emitters, using a calibrated sphere, fiber coupled to a compact array spectroradiometer traceable to the National Research Council. The emitting samples used in the measurements were prepared in the form of packed powder inside a $900-\mu$ m-diameter, 3-mm-deep cylinder at the center of a 2.5-cm-diameter acrylic hemisphere.

The observations described in the case of Yb, Tm:YLF regarding its temperature behavior as a function of pumping conditions apply directly to a second blue up-conversion material that is more suitable for our UC display application: $\mathrm{Yb}$, Tm-doped potassium tri-yttrium decafluoride or $\mathrm{KY}_{3} \mathrm{~F}_{10}$ (Ref. 15) which happens to be most efficiently excited at 
TABLE II. Absolute up-conversion efficiencies measured with the best performing, up-conversion candidates for red, green, and blue emission excited with a 976-nm, 30-Hz, 2-ms pulsed excitation. The powder was packed in a 900- $\mu \mathrm{m}$-diameter, 3-mm-deep hole inside an acrylic hemisphere of 2.5 -cm diameter. The estimated temperature reached by the emitting powder at the listed efficiency is indicated.

\begin{tabular}{|c|c|c|c|}
\hline \multirow[b]{2}{*}{ Emitter } & \multicolumn{2}{|c|}{$\begin{array}{l}\text { Pulsed excitation, no thermal management } \\
\text { maximum measured raw efficiency }\end{array}$} & \multirow{2}{*}{$\begin{array}{l}\text { Temperature } \\
\text { reached at the listed efficiency } \\
\left({ }^{\circ} \mathrm{C}\right)\end{array}$} \\
\hline & $(\%)$ & $(1 \mathrm{~m} / \mathrm{W})$ & \\
\hline $\begin{array}{c}\text { Red } \\
\left(\mathrm{Yb}, \mathrm{Er}: \mathrm{YF}_{3}\right)\end{array}$ & 7.8 & 3.6 & 25 \\
\hline $\begin{array}{c}\text { Green } \\
(\mathrm{Yb}, \mathrm{Er}: \mathrm{NYF})\end{array}$ & 5.8 & 37.5 & 25 \\
\hline $\begin{array}{c}\text { Blue } \\
\left(\mathrm{Yb}, \mathrm{Tm}: \mathrm{KY}_{3} \mathrm{~F}_{10}\right)\end{array}$ & 4.5 & 3.35 & 120 \\
\hline
\end{tabular}

$974.7 \mathrm{~nm}$. This is very close to the optimized excitation wavelength for the green and red emitters, so the use of the blue up converter simplifies the design of the display and avoids the need for an uncommon 960-nm diode laser. Furthermore, $\mathrm{Yb}, \mathrm{Tm}: \mathrm{KY}_{3} \mathrm{~F}_{10}$ is more efficient than Yb,Tm:YLF. The data for maximum efficiency achieved with the materials used were taken with $30-\mathrm{Hz}, 2-\mathrm{ms}$ duration pulsed excitation and are listed in Table II. The temperature reached with the blue up-conversion emitter was $120^{\circ} \mathrm{C}$, which means that even higher efficiency could be achieved by using an emitter configured for thermal management such as one with a heat diffusing substrate and smaller pixels.

\section{CONCLUSIONS}

The role of temperature on the up conversion of infrared light to visible has been investigated experimentally and a rate equation model that includes temperature-dependent energy-transfer coefficients was developed. The model agrees well with the experimental observations and illustrates the critical role played by temperature in the blue upconversion efficiency. Several means to reduce the operating temperature were presented such as using short-pulse excitation, using a heat conductive substrate, and designing pixel shapes for optimal heat extraction. The absolute efficiencies of three primary-color up-conversion materials candidates for optically written displays were measured and are presented.

\footnotetext{
${ }^{1}$ N. Bloembergen, Phys. Rev. Lett. 2, 84 (1959).

${ }^{2}$ F. Auzel, Ann. Telecommun. 24, 363 (1969).

${ }^{3}$ J. E. Geusic, F. W. Ostermayer, H. M. Marcos, L. G. Van Uitert, and J. P. van der Ziel, J. Appl. Phys. 42, 1958 (1971).

${ }^{4}$ H. J. Guggenheim and L. F. Johnson, Appl. Phys. Lett. 15, 51 (1969).
}

${ }^{5}$ L. F. Johnson, J. E. Geusic, H. J. Guggenheim, T. Kushida, S. Singh, and L. G. Van Uitert, Appl. Phys. Lett. 15, 48 (1969).

${ }^{6}$ L. G. Van Uitert, S. Singh, H. J. Levinstein, L. F. Johnson, W. H. Grodkiewicz, and J. E. Geusic, Appl. Phys. Lett. 15, 53 (1969).

${ }^{7}$ L. Esterowitz, A. Schnitzler, J. Noonan, and J. Bahler, Appl. Opt. 7, 2053 (1968).

${ }^{8}$ A. Rapaport, J. Milliez, A. Cassanho, H. Jenssen, and M. Bass, Opt. Express 12, 5215 (2004).

${ }^{9}$ A. Rapaport, J. Milliez, F. Szipocs, H. Jenssen, A. Cassanho, and M. Bass, SID'2003 International Symposium-Digest of Technical Papers, Baltimore, MD, USA, 18-23 May 2003 (Society of International Display, San Jose, CA, 2003), pp. 1230-1233.

${ }^{10}$ A. Rapaport, F. Szipocs, J. Milliez, H. Jenssen, M. A. Bass, K. J. Schafer, and K. D. Belfield, Proc. SPIE 4294, 47 (2001).

${ }^{11}$ A. Rapaport, F. Szipocs, J. Milliez, H. Jenssen, M. Bass, K. Schafer, K. Belfield, and M. Bass, IDW'00. Proceedings of the Seventh International Display Workshops, Kobe, Japan, 29 November-1 December 2005 (Society of International Display, San Jose, CA, 2000), pp. 825-828.

${ }^{12}$ A. Rapaport, J. Milliez, H. Jenssen, A. Cassanho, and M. Bass, SID’2004 International Symposium-Digest of Technical Papers, Seattle, WA, USA, 23-28 May 2004 (Society of International Display, San Jose, CA, 2004), pp. 1237-1239.

${ }^{13}$ G. Huber, E. Heumann, T. Sandrock, and K. Petermann, J. Lumin. 72-74, 1 (1997).

${ }^{14}$ F. Auzel, Phys. Rev. B 13, 2809 (1976).

${ }^{15}$ A. Rapaport, J. Milliez, F. Szipocs, A. Cassanho, H. Jenssen, and M. Bass, Appl. Opt. 43, 6477 (2004).

${ }^{16}$ M. Bass, Physical Processes in Laser-Materials Interactions, Proceedings of the 1980 NATO Advanced Study Institute, 1983 (unpublished), pp. $77-115$.

${ }^{17}$ See, for example, http://www.tydex.ru/materials/materials5/ylf.html

${ }^{18}$ H. Berthou and C. K. Jorgensen, Opt. Lett. 15, 1100 (1990).

${ }^{19}$ P. V. Dos Santos, M. T. De Araujo, A. S. Gouveia-Neto, J. A. M. Neto, and A. S. B. Sombra, IEEE J. Quantum Electron. 35, 395 (1999).

${ }^{20}$ J. L. Sommerdijk, J. Lumin. 4, 441 (1971).

${ }^{21}$ A. Diening, P. E. A. Mobert, and G. Huber, J. Appl. Phys. 84, 5900 (1998).

${ }^{22}$ B. M. Walsh, N. P. Barnes, and B. Di Bartolo, J. Appl. Phys. 83, 2772 (1998).

${ }^{23}$ T. Riedener, H. U. Guedel, G. C. Valley, and R. A. McFarlane, J. Lumin. 63, 327 (1995). 